\title{
Urban Wild Boars and Risk for Zoonotic Streptococcus suis, Spain
}

\section{Xavier Fernández-Aguilar, Marcelo Gottschalk, Virginia Aragon, Jordi Càmara, Carmen Ardanuy, Roser Velarde, Nuria Galofré-Milà, Raquel Castillo-Contreras, Jorge R. López-Olvera, Gregorio Mentaberre, Andreu Colom-Cadena, Santiago Lavín, Oscar Cabezón}

Urban wild boars (Sus scrofa) from Barcelona, Spain, harbor great diversity of Streptococcus suis strains, including strains with the cps2 gene and with the same molecular profile as local human cases. The increasing trend of potential effective contacts for S. suis transmission is of public health concern.

$\mathrm{P}$ opulations of the European wild boar (Sus scrofa) have been increasing (1). The wild boar's high adaptability to human-dominated landscapes and its increase in human tolerance have also prompted its presence in urban areas, leading to conflicts with local humans $(2,3)$. Health risk assessment should therefore be performed in this new scenario, in which wild boars and their interactions with humans are becoming common in heavily populated areas (3).

Streptococcus suis is an emerging zoonotic pathogen (4). Among the bacterium's 35 described serotypes, serotypes 2,5 , and 14 are those most related to human disease $(4,5)$. Several risk factors have been associated with $S$. suis infection in humans, such as consumption of undercooked pig meat or work in pig-related occupations (5). The wild boar has also been identified as a source of human infection in relation to hunting activities (6). However, the increasing presence of synanthropic wild boars may also pose a novel public health risk for nonhunters. The objective of

Author affiliations: Centre de Recerca en Sanitat Animal (CReSA, IRTA-UAB), Bellaterra, Spain (X. Fernández-Aguilar, V. Aragon,

N. Galofré-Milà, O. Cabezón); Universitat Autònoma de

Barcelona, Bellaterra (X. Fernández-Aguilar, R. Velarde,

R. Castillo-Contreras, J.R. López-Olvera, G. Mentaberre,

A. Colom-Cadena, S. Lavín, O. Cabezón); Université de Montréal,

St.-Hyacinthe, Québec, Canada (M. Gottschalk); Hospital

Universitari de Bellvitge, L'Hospitalet de Llobregat, Spain

(J. Càmara, C. Ardanuy); CIBER de Enfermedades Respiratorias, Madrid, Spain (C. Ardanuy)

DOI: https://doi.org/10.3201/eid2406.171271 this study was to investigate the presence of $S$. suis strains with zoonotic potential in wild boars from the metropolitan area of Barcelona, Spain, and assess the risk of transmission to humans.

\section{The Study}

The metropolitan area of Barcelona is heavily populated (>3.2 million) and includes Collserola Natural Park, 8,000 hectares of Mediterranean forest (Figure 1). The wild boar population in this forest is $\approx 1,200$, and habituation to humans has become common (2). A total of 108 apparently healthy wild boars were sampled in the Barcelona area for the presence of S. suis during April-November 2015; the animals had been hunted in regular campaigns, captured by box traps and euthanized for population control, or captured with the aid of anesthetic darts and euthanized because of public risk. All procedures were performed under the regulation of the competent public administrations and in compliance with current guidelines for ethical use of animals in research, following European (2010/63/EU) and Spanish (R.D. 53/2013) legislation.

At postmortem examination, we collected tonsil and nasal swab specimens from each wild boar and cultured them on chocolate agar. For each sample, a maximum of 4 presumptive $S$. suis colonies were subcultured and subsequently identified with a $S$. suis-specific gene recN PCR (7). We molecularly serotyped Streptococcus suis isolates (8) and further serotyped positive isolates for $c p s 2$ gene by coagglutination (9). We also included 2 local S. suis serotype 2 strains of human origin for molecular characterization (Table 1). The source of the infection was not identified in 1 of these 2 patients (10), but the second patient acquired the infection through an accidental cut on his hand with a tusk while manipulating a wild boar hunted $50 \mathrm{~km}$ from Barcelona. In the days immediately following his injury, this patient developed pain in his hand; 5 days after the injury, he sought care at an emergency department. He had fever (body temperature $\left.39.5^{\circ} \mathrm{C}\right)$, leukocytosis $\left(35 \times 10^{9}\right.$ cells $/ \mathrm{L}$; reference range 3.9-10 $\times 10^{9}$ cells/L), limited arm mobility, headache, and lethargic mental status. At this stage, the patient received diagnoses of arthritis and meningitis.

We isolated S. suis from 91 (84.3\%; 95\% CI 76.2\%$89.9 \%$ ) wild boars, identifying 332 isolates from tonsil $(\mathrm{n}=141)$ and nasal $(\mathrm{n}=191)$ swab specimens. We found 




Figure 1. Part of the metropolitan area of Barcelona, Spain, showing land characteristics, Collserola Natural Park, the location of the wild boars sampled, and results of Streptococcus suis serotype 2 strains, identified by both isolation and molecular detection. Letters indicate locations where several wild boars were sampled, obtained by box traps $(A, n=21)$ or regular hunting campaigns $(B, n=9$; $C, n=14 ; D, n=5)$.

17 different serotypes and nontypeable strains in the wild boar population (Table 2). Three of the colonies were serotype 2 , isolated from 2 wild boars, and were confirmed along with the human strains as serotype 2 by coagglutination test. We further characterized serotype 2 strains (both wild boar and human origin) using multilocus sequence typing (11) and by assessing the presence of the genes muramidase-released protein ( $m r p$ ), extracellular factor (ef), and hemolysin suilysin (sly), associated with invasiveness and zoonotic potential (12). These serotype 2 strains belonged to clonal complex 1 and had the same molecular characterization (sequence type [ST] 1; cps $2 /$ $m r p+/ e f+/ s l y+$ ), with the exception of 1 human isolate that was ST3 (a single-locus variant of ST1) (Table 1). Results from enterobacterial repetitive intergenic consensus (ERIC) PCR (13) showed a unique fingerprint profile for all serotype 2 strains and 32 different fingerprints for 36 other isolates (originating from 10 wild boars). In addition, 4 of 94 wild boars $(4.3 \%$; $95 \%$ CI $1.7 \%-10.4 \%)$ tested positive for detection of the cps 2 gene directly in nasal swab specimens.
To evaluate the occurrence and trend of potential effective contacts between urban wild boars and humans, we examined 174 veterinary field interventions related to wild boar incidents in Barcelona during 2013-2016. We found that both the number of interventions and the percentage of interventions motivated by violent physical contacts or aggressive interactions with humans (charges, bites, or injuries) showed an increasing trend (Figure 2).

\section{Discussion}

The isolation of S. suis in apparently healthy wild boars from the metropolitan area of Barcelona highlights its reservoir role and identifies this species as a potential source for human infections in urban areas. The high number of wild boars carrying S. suis confirms results obtained in a previous study, in which $S$. suis and $S$. suis-like strains were detected together through the use of a PCR for the $g d h$ gene (14). Our results show a high diversity of $S$. suis serotypes, with and without pathogenic relevance for humans (4). A high percentage of isolates was nontypeable, as previously found in both domestic pig and wild boar carriers (4). These results

Table 1. Sequence type and virulence associated gene profile of the Streptococcus suis serotype 2 strains isolated from wild boars and humans in metropolitan area of Barcelona, Spain, 2012-2015*

\begin{tabular}{|c|c|c|c|c|c|c|c|}
\hline \multirow[b]{2}{*}{ Source } & \multirow[b]{2}{*}{ Year } & \multirow[b]{2}{*}{ Clinical infection } & \multirow[b]{2}{*}{ MLST } & \multicolumn{3}{|c|}{ Virulence genes } & \multirow[b]{2}{*}{ References } \\
\hline & & & & $m r p$ & ef & sly & \\
\hline Human male, 57 y of age & 2012 & Meningitis, arthritis, bacteremia & ST3 & + & + & + & $(10)$ \\
\hline Human male, 48 y of age & 2014 & Meningitis, arthritis & ST1 & + & + & + & This study \\
\hline Wild boar & 2015 & No & ST1 & + & + & + & This study \\
\hline Wild boar & 2015 & No & ST1 & + & + & + & This study \\
\hline Wild boar & 2015 & No & ST1 & + & + & + & This study \\
\hline
\end{tabular}

*MLST, multilocus sequence typing; ST, sequence type. 
Table 2. Frequency of Streptococcus suis serotypes identified by multiplex PCR in isolates from 108 wild boars from the metropolitan area of Barcelona, Spain, 2012-2015*

\begin{tabular}{|c|c|c|c|c|c|c|}
\hline \multirow[b]{2}{*}{ Serotype } & \multicolumn{4}{|c|}{ Isolates } & \multicolumn{2}{|c|}{ Wild boar } \\
\hline & $\begin{array}{c}\text { No. nasal samples } \\
\text { positive }\end{array}$ & $\begin{array}{c}\text { No. tonsillar } \\
\text { samples positive }\end{array}$ & $\begin{array}{c}\text { Total no. samples } \\
\text { positive }\end{array}$ & $\begin{array}{c}\text { Prevalence, \% } \\
(95 \% \mathrm{Cl})\end{array}$ & $\begin{array}{c}\text { No. animals } \\
\text { positive* }\end{array}$ & $\begin{array}{c}\text { Prevalence \% } \\
(95 \% \mathrm{Cl})\end{array}$ \\
\hline 2 & 2 & 1 & 3 & $0.9(0.3-2.6)$ & 2 & $1.8(0.5-6.5)$ \\
\hline 4 & 3 & 7 & 10 & $3.0(1.6-5.4)$ & 10 & $9.2(5.1-16.2)$ \\
\hline 6 & 5 & 0 & 5 & $1.5(0.6-3.5)$ & 2 & $1.8(0.5-6.5)$ \\
\hline 7 & 0 & 2 & 2 & $0.6(0.2-2.2)$ & 2 & $1.8(0.5-6.5)$ \\
\hline 8 & 3 & 1 & 4 & $1.2(0.5-3.1)$ & 3 & $2.8(0.9-7.8)$ \\
\hline 9 & 7 & 18 & 25 & $7.5(5.1-10.9)$ & 13 & $12.0(7.2-19.5)$ \\
\hline 10 & 4 & 1 & 5 & $1.5(0.6-3.5)$ & 3 & $2.8(0.9-7.8)$ \\
\hline 12 & 0 & 1 & 1 & $0.3(0.0-1.7)$ & 1 & $0.9(0.0-5.1)$ \\
\hline 15 & 0 & 4 & 4 & $1.2(0.5-3.1)$ & 3 & $2.8(0.9-7.8)$ \\
\hline 16 & 27 & 7 & 34 & $10.2(7.4-14.0)$ & 17 & $15.7(10.1-23.8)$ \\
\hline 17 & 1 & 0 & 1 & $0.3(0.0-1.7)$ & 1 & $0.9(0.0-5.1)$ \\
\hline 21 & 30 & 0 & 30 & $9.0(6.4-12.6)$ & 12 & $11.1(6.5-18.4)$ \\
\hline 23 & 3 & 0 & 3 & $0.9(0.3-2.6)$ & 3 & $2.8(0.9-7.8)$ \\
\hline 27 & 2 & 2 & 4 & $1.2(0.5-3.1)$ & 4 & $3.7(1.4-9.1)$ \\
\hline 28 & 1 & 2 & 3 & $0.9(0.3-2.6)$ & 3 & $2.8(0.9-7.8)$ \\
\hline 31 & 20 & 13 & 33 & $9.9(7.1-13.6)$ & 23 & $21.3(14.6-29.9)$ \\
\hline 33 & 3 & 0 & 3 & $0.9(0.3-2.6)$ & 2 & $1.8(0.5-6.5)$ \\
\hline Nontypeable & 80 & 82 & 162 & $48.8(43.5-54.1)$ & 70 & $64.8(55.4-73.2)$ \\
\hline Total & 191 & 141 & 332 & 100 & $108 \dagger$ & 100 \\
\hline
\end{tabular}

further suggest that $S$. suis and serotype 2 strains are widely distributed in European wild boar populations (14).

Most human disease cases reported worldwide are associated with invasive serotype 2 strains that typically cause meningitis and, ultimately, neurologic sequelae or death $(4,5)$. ST1, belonging to clonal complex 1 , is one of the most frequent sequence types found in human and pig disease cases from Europe and other regions; it often involves the encoding virulence genes $m r p$, ef, and sly found in cps2-positive wild boar strains (15). Despite the high diversity of genomic fingerprints found in wild boar isolates, a unique fingerprint included all

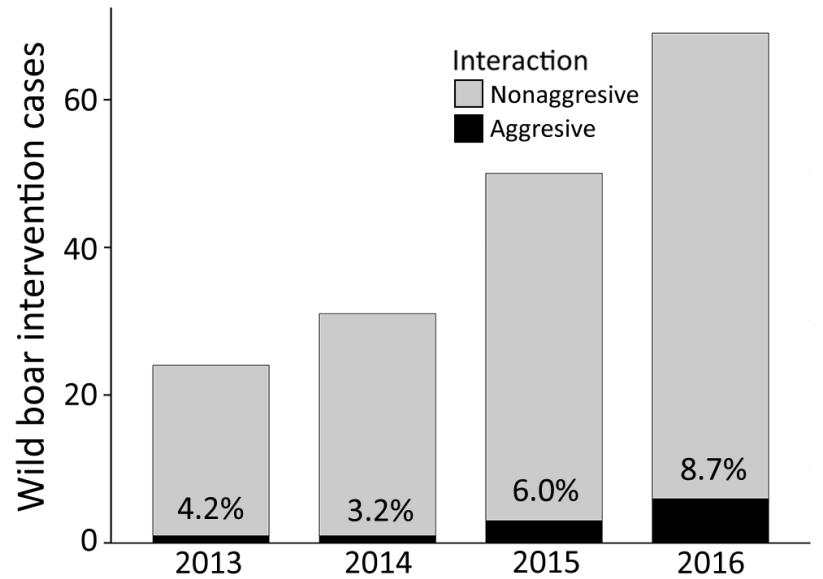

Figure 2. Occurrence and temporal trend of veterinary interventions related to wild boar removal in Barcelona, Spain, 2013-2016, and percentage of interventions with aggressive interactions. Aggressive interaction involves both violent physical contacts (charging or pushing for food) and aggressions to humans (bites). serotype 2 strains, suggesting no consistent differences between human and wild boar isolates. However, wild boars harboring cps2-positive strains were apparently healthy carriers. Other serotypes found in apparently healthy wild boars, such as $5,9,14,16$, and 21 , have also been occasionally associated with severe human disease $(4,5)$.

At least 7 human cases of wild boar bites were treated at the emergency department of a main local hospital (2012-2015, Communication Unit of Vall d'Hebron, Barcelona), yet small wounds not attended in health centers can be enough to acquire systemic infections (4). Human $S$. suis infections are associated mainly with pig farming and the food industry, being considered an occupational disease or foodborne disease in developing countries (5). The increasing trend of potential effective contacts for $S$. suis transmission between urban wild boars and humans in Barcelona suggests that $S$. suis can be acquired by humans who belong to apparently lowrisk collectives.

\section{Conclusions}

Wild boars from the metropolitan area of Barcelona harbor $S$. suis strains with the same enterobacterial repetitive intergenic consensus PCR fingerprints and virulence gene profiles as invasive strains of local human origin; thus, they have zoonotic and invasive potential. The increasing interactions of wild boars with humans in urban areas pose risks for $S$. suis or other directly or indirectly transmitted zoonoses, making the presence of urban wild boars a public health concern. 


\section{Acknowledgments}

The authors are indebted to the Generalitat de Catalunya (Serveis Territorials d'Agricultura, Ramaderia, Pesca i Alimentació a Barcelona) and the Parc Natural de Collserola for their collaboration. The authors are also grateful for the technical contribution of Sonia Lacouture from the Diagnostic Service of the University of Montreal and to the colleagues from Servei d'Ecopatologia de Fauna Salvatge who contributed to the wild boar sample collection.

This research benefited from contracts $13 / 051$ and 15/0174 between the Universitat Autònoma de Barcelona and the Ajuntament de Barcelona. This study was also supported by Centro de Investigación Biomédica en Red (CIBER) de Enfermedades Respiratorias (CIBERES CB06/06/0037), an initiative of the Instituto de Salud Carlos III, Madrid, Spain. Xavier Fernández-Aguilar, Andreu Colom-Cadena, and Raquel Castillo-Contreras were supported by a PhD grant FI-DGR co-financed by Generalitat de Catalunya and European Social Fund (ESF).

\section{About the Author}

Dr. Fernández-Aguilar is a researcher in the Wildlife Ecology and Health Group and the Servei d'Ecopatologia de Fauna Salvatge, Departament de Medicina i Cirurgia Animals, Universitat Autònoma de Barcelona, Spain. His main research interests are the ecology of infectious diseases at the wildlife-livestock interface and disease dynamics in wild animal populations.

\section{References}

1. Massei G, Kindberg J, Licoppe A, Gačić D, Šprem N, Kamler J, et al. Wild boar populations up, numbers of hunters down? A review of trends and implications for Europe. Pest Manag Sci. 2015;71:492-500. http://dx.doi.org/10.1002/ps.3965

2. Castillo-Contreras R, Carvalho J, Serrano E, Mentaberre G, Fernández-Aguilar X, Colom A, et al. Urban wild boars prefer fragmented areas with food resources near natural corridors. Sci Total Environ. 2018;615:282-8. http://dx.doi.org/10.1016/ j.scitotenv.2017.09.277

3. Jansen A, Luge E, Guerra B, Wittschen P, Gruber AD, Loddenkemper C, et al. Leptospirosis in urban wild boars, Berlin, Germany. Emerg Infect Dis. 2007;13:739-42. http://dx.doi.org/ 10.3201/eid1305.061302

4. Goyette-Desjardins G, Auger J-P, Xu J, Segura M, Gottschalk M. Streptococcus suis, an important pig pathogen and emerging zoonotic agent - an update on the worldwide distribution based on serotyping and sequence typing. Emerg Microbes Infect. 2014;3:e45. http://dx.doi.org/10.1038/emi.2014.45

5. Huong VTL, Ha N, Huy NT, Horby P, Nghia HDT, Thiem VD, et al. Epidemiology, clinical manifestations, and outcomes of Streptococcus suis infection in humans. Emerg Infect Dis. 2014;20:1105-14. http://dx.doi.org/10.3201/eid2007.131594

6. Rosenkranz M, Elsner H-A, Stürenburg HJ, Weiller C, Röther J, Sobottka I. Streptococcus suis meningitis and septicemia contracted from a wild boar in Germany. J Neurol. 2003;250:869-70. http://dx.doi.org/10.1007/s00415-003-1103-3

7. Ishida S, Tien HT, Osawa R, Tohya M, Nomoto R, Kawamura Y, et al. Development of an appropriate PCR system for the reclassification of Streptococcus suis. J Microbiol Methods. 2014;107:66-70. http://dx.doi.org/10.1016/j.mimet.2014.09.003

8. Okura M, Lachance C, Osaki M, Sekizaki T, Maruyama F, Nozawa T, et al. Development of a two-step multiplex PCR assay for typing of capsular polysaccharide synthesis gene clusters of Streptococcus suis. J Clin Microbiol. 2014;52:1714-9. http://dx.doi.org/10.1128/JCM.03411-13

9. Gottschalk M, Higgins R, Boudreau M. Use of polyvalent coagglutination reagents for serotyping of Streptococcus suis. J Clin Microbiol. 1993;31:2192-4.

10. Gómez-Zorrilla S, Ardanuy C, Lora-Tamayo J, Cámara J, García-Somoza D, Peña C, et al. Streptococcus suis infection and malignancy in man, Spain. Emerg Infect Dis. 2014;20:1067-8. http://dx.doi.org/10.3201/eid2006.131167

11. King SJ, Leigh JA, Heath PJ, Luque I, Tarradas C, Dowson CG, et al. Development of a multilocus sequence typing scheme for the pig pathogen Streptococcus suis: identification of virulent clones and potential capsular serotype exchange. J Clin Microbiol. 2002;40:3671-80. http://dx.doi.org/10.1128/ JCM.40.10.3671-3680.2002

12. Silva LMG, Baums CG, Rehm T, Wisselink HJ, Goethe R, Valentin-Weigand P. Virulence-associated gene profiling of Streptococcus suis isolates by PCR. Vet Microbiol. 2006;115:11727. http://dx.doi.org/10.1016/j.vetmic.2005.12.013

13. Versalovic J, Koeuth T, Lupski JR. Distribution of repetitive DNA sequences in eubacteria and application to fingerprinting of bacterial genomes. Nucleic Acids Res. 1991;19:6823-31. http://dx.doi.org/10.1093/nar/19.24.6823

14. Baums CG, Verkühlen GJ, Rehm T, Silva LMG, Beyerbach M, Pohlmeyer K, et al. Prevalence of Streptococcus suis genotypes in wild boars of northwestern Germany. Appl Environ Microbiol. 2007;73:711-7. http://dx.doi.org/10.1128/ AEM.01800-06

15. Fittipaldi N, Segura M, Grenier D, Gottschalk M. Virulence factors involved in the pathogenesis of the infection caused by the swine pathogen and zoonotic agent Streptococcus suis. Future Microbiol. 2012;7:259-79. http://dx.doi.org/10.2217/fmb.11.149

Address for correspondence: Xavier Fernández-Aguilar,

Universitat Autònoma de Barcelona, Medicina i Cirurgia Animals, Faculty of Veterinary Medicine, Edifici V, Bellaterra, 08193 Spain; email: xfdezaguilar@gmail.com 